\title{
Some Possible Particles Decays from $p p$ Collisions at LHC Experiment
}

\author{
Hasan Arslan \\ Physics Department, Bingöl University, Bingöl, Turkey \\ Email: hasanarslan46@yahoo.com
}

Received 29 February 2014; revised 22 April 2014; accepted 14 May 2014

Copyright (C) 2014 by author and Scientific Research Publishing Inc.

This work is licensed under the Creative Commons Attribution International License (CC BY).

http://creativecommons.org/licenses/by/4.0/

(c) (i) Open Access

\begin{abstract}
Some of the possible decays of $p p$ collisions at LHC experiment are considered. The vector bosons mediating in the electroweak interactions and right-handed leptons except neutrinos are assumed to be the most resultant particles from the pp collisions. Neutrinos and anti-neutrinos will be observed when one-double electron charged vector bosons are the resultant particles. The charge conservation is thought to be the dominant factor of these decays. The amplitude transitions for Feynman diagram of these decays are written.
\end{abstract}

Keywords

$Z^{\prime}$ Boson, $W^{\prime}$ Bosons, Resultant Particles from $p p$ Collisions, Scattering Amplitude

\section{Introduction}

The Standard Model (SM) is in difficulties with defining the problems:

1) The Higgs particle mass,

2) Including the gravitational interactions to the SM,

3) The dark matter representation in the model,

4) The masses for neutrinos,

5) Large gap energy between electroweak scale and Planck scale,

6) The unification of gauge couplings of the electroweak interactions at some energy scale if the four fundamental forces are the result of a local gauge theory with a higher fundamental symmetry.

The recent experiments mainly LHC and the future colliders will solve these problems. The electromagnetic field interactions and weak interactions are combined in a single representation by the Glashow-Weinberg-Salam Model of the electroweak interactions based on the gauge group $\mathrm{SU}(2) \times \mathrm{U}(1)$ which is in good agreement 
with the experimental results. Later the strong interactions are included to the theory and the group is written as $\mathrm{SU}(3) \mathrm{C} \times \mathrm{SU}(2) \mathrm{L} \times \mathrm{U}(1) \mathrm{Y}$. This group defines the SM particles. Since the four fundamental forces; weak, electromagnetic, strong, and gravity; are equal at the Grand Unified Theory (GUT) energy scale, the some of the additional neutral gauge bosons might be discovered at CERN LHC experiment as well as the lightest supersymmetric stable particle of the dark energy and the particles which have at the same time the properties of the leptons and quarks or the states changes to each other named as lepto-quarks or exotic fermions. The fermionic particles of the SM are grouped in the representation of the three generations as

$$
\left(\begin{array}{c}
v_{e} \\
e \\
u \\
d
\end{array}\right),\left(\begin{array}{c}
v_{\mu} \\
\mu \\
c \\
s
\end{array}\right),\left(\begin{array}{c}
v_{\tau} \\
\tau \\
t \\
b
\end{array}\right)
$$

The first two particles of each group are the leptons with charges $0,-1$ respectively in the unit of the electron charge. The last two are quarks with the electron charge $+2 / 3$ and $-1 / 3$ for $u$ and $d$ type quarks. Each particles described have their anti-particles. These particles all together build up the matter around us.

\section{Some Particles Decaying from $p p$ Collisions at LHC}

The breaking of $E_{6}$ gives other gauge groups. Concentrating on the $\mathrm{SU}(2) \mathrm{L} \times \mathrm{U}(1) \mathrm{Y} \times \mathrm{U}(1)$ effective theory, the $U(1)_{\chi}, U(1)_{\psi}, U(1)_{\eta}$ are the possible U(1)'s in broken E6 GUT's and the extra U(1) is an Abelian symmetry with its associated "hypercharge" $\mathrm{Y}[1]$;

$$
\begin{aligned}
& E_{6} \rightarrow S O(10) \times U(1)_{\psi} \\
& S O \rightarrow S U(5) \times U(1)_{\chi} \\
& E_{6} \rightarrow S U(3) \times S U(2) \times U(1) \times U(1)_{\eta}
\end{aligned}
$$

The symmetry will be spontaneously broken by a Higgs sector consisting of one doublet and one singlet [1].

The decays $p p \rightarrow W^{\prime} \rightarrow \overline{l l}$ have the same validity as the decays given by $p p \rightarrow Z^{\prime} \rightarrow \overline{l l}$, where $\bar{l}$ refers to anti-leptons except right-handed neutrinos. This is true because in [2] [3] the mass of the $Z^{\prime}$ is estimated by calculating the $Z^{\prime}$ decay width as the same procedure of calculating the $Z^{0}$ boson decay width. Using the relation [4]

$$
M_{Z} \rightarrow M_{W} / \cos \theta_{W}
$$

given for the $Z^{\prime}$ and $W^{\prime}$, there is no doubt in writing the relation as

$$
M_{W^{\prime}} \rightarrow M_{Z^{\prime}} \cos \theta_{W} .
$$

In [2] [3] $Z^{\prime}$ mass is estimated around $630 \mathrm{GeV}$ and the Weinberg angle is taken as

$$
\sin ^{2} \theta_{w}=\frac{3}{8}
$$

at GUT scale. Therefore,

$$
M_{W^{\prime}} \approx 498 \mathrm{GeV} .
$$

Using the conservation of the protons' charges, some of the possible $p p$ collisions decays will be such that (Figure 1 and Figure 2).

When it is assumed that the anti-leptons are the resultant particles as the vector bosons then the right-handed neutrinos couldn't be seen as the resultant particles. In these decays instead of the intermediate vector bosons the neutral and plus-charged Higgs bosons could take the place. The Higgs bosons would be the plus ones replaced in the resultant particles such that $\overline{l l}$. The neutral Higgs boson could be the one replaced in neutral mediating vector bosons $Z^{\prime}, W^{\prime}$.

The scattering amplitude for the diagrams in Figure 1 and Figure 2 can be written down by using the amplitude evaluated for the $\pi^{+} K^{+}$scattering in [5] as 


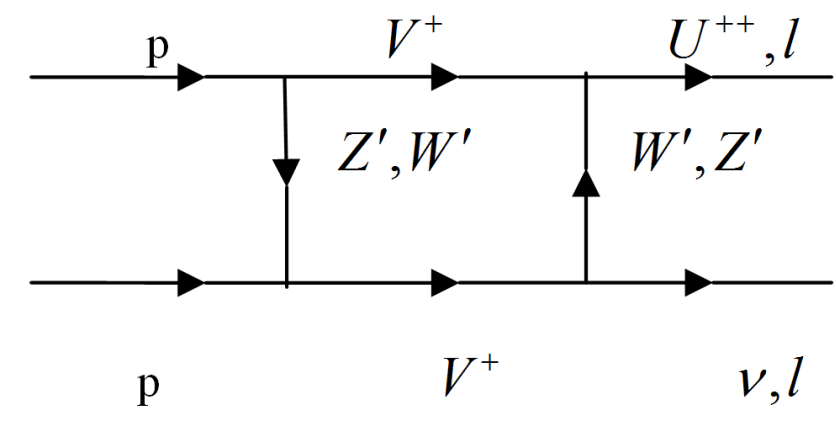

Figure 1. The diagram for the decay $p p \rightarrow U^{++} v, \overline{l l}$.

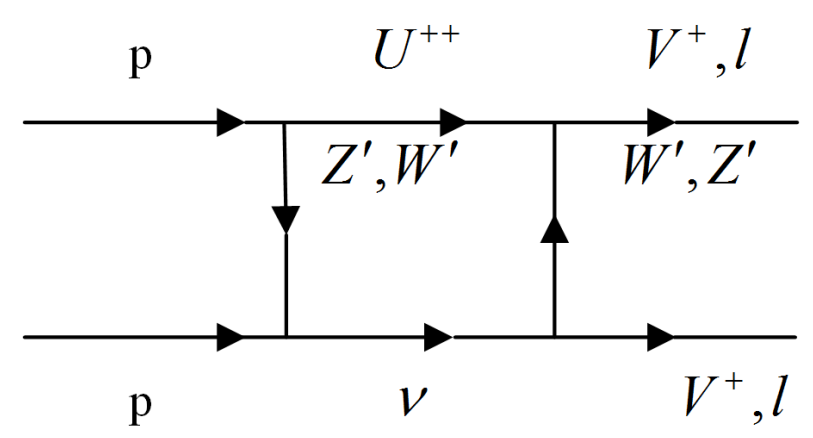

Figure 2. The diagram for the decay $p p \rightarrow V^{+} V^{+}, \overline{l l}$.

$$
\begin{aligned}
& S_{f i}^{1}\left(p p \rightarrow V^{+} V^{+}, \overline{l l}, U^{++} v, \pi^{+} \pi^{+}\right) \\
& =-i \int \mathrm{d}^{4} x J_{\mu}(p) A^{\mu}\left(V^{+}\right)
\end{aligned}
$$

where

$$
A^{\mu}\left(V^{+}\right)=-\frac{1}{q^{2}} J^{\mu}\left(V^{+}\right)=-\frac{1}{q^{2}} e N_{3} N_{4}\left(p_{V_{1}}+p_{V_{2}}\right) \mathrm{e}^{i q \cdot x}
$$

with

$$
q=p_{V_{2}}-p_{V_{1}}
$$

and $p_{V_{2}}, p_{V_{1}}$ are the four momentum of the resultant particles. The 4-curent for proton is:

$$
J_{\mu}(p)=e N_{1} N_{2}\left(p_{1}+p_{2}\right)_{\mu} .
$$

Since the protons have the same 4-momentum it is true to write down:

$$
p_{1}=p_{2}=p
$$

Therefore, the exponential term not written in Eqn. (10) is 1. The scattering amplitude can be written as

$$
S_{f i}^{1}=-2 e^{2} N^{2} N_{3} N_{4}(2 \pi)^{4} \delta^{4}\left(p_{V_{2}}+p_{V_{1}}-2 p\right) p_{\mu} x\left(-i \frac{g^{\mu \nu}}{q^{2}}\right)\left(p_{V_{2}}+p_{V_{1}}\right)
$$

Again the waves for both of two protons must be the same, so $N_{1}=N_{2}=N$ is taken in Equation (12). In Equation (12) the $N, N_{3}$, and $N_{4}$ are the normalization factors; $-\frac{i g^{\mu \nu}}{q^{2}}$ is the propagator for the mediating bosons; $q$ refers to the momentum differences between the final and the initial states, and the four-momenta conservation is given by the term $(2 \pi)^{4} \delta^{4}\left(p_{V_{2}}+p_{V_{1}}-2 p\right)$ for incoming and outgoing particles. 


\section{Conclusion}

Although the SM has some difficulties, it is strongly a correct model to extend at the GUT scale. By using the previous works the $W^{\top}$ gauge boson mass is estimated to be around $498 \mathrm{GeV}$. The resultant particles from $p p$ collisions are assumed to be a double positive-electron charged vector boson with a neutrino or anti-neutrino, or, two vector bosons each with one positive-electron charge, instead of the latter one; two anti-leptons are also accepted except the neutrinos. The $p p$ collisions at GUT scale will give the scattering amplitudes as done in this work.

\section{Acknowledgements}

I would like to thank to Prof. Dr. Durmuş Ali Demir for his opinions in studying this subject.

\section{References}

[1] Dib, C. and Gilman, J.F. (1987) Physical Review D, 36, 1337-1343. http://dx.doi.org/10.1103/PhysRevD.36.1337

[2] Arslan, H. (2008) Extra Gauge Bosons in E6 Models, Ph.D. Thesis, Çukurova University, Adana.

[3] Aydemir, A., Arslan, H. and Topaksu, A.K. (2009) Physics of Particles and Nuclei Letters, 6, 496-503. http://dx.doi.org/10.1134/S1547477109040049

[4] Aitchison, I.J.R. and Hey, A.J.G. (1982) Gauge Theories in Particle Physics. Adam Hilger, London.

[5] Greiner, W., Schramm, S. and Stein, E. (2002) Quantum Chromodynamics. Springer-Verlag, Berlin, Heidelberg. 